\title{
Challenges in the development of an HIV-1 vaccine
}

\author{
Dan H. Barouch ${ }^{1}$
}

The development of a safe and effective human immunodeficiency virus (HIV)-1 vaccine is a critically important global health priority. Despite recent advances in our understanding of HIV-1 pathogenesis and immunology, however, major scientific obstacles remain. Prototype HIV-1 vaccine candidates aimed at eliciting humoral and cellular immune responses have so far failed to protect against HIV-1 infection or to reduce viral loads after infection in clinical efficacy studies. A renewed and coordinated commitment to basic discovery research, preclinical studies and clinical trials will therefore be required to overcome the hurdles currently facing the field. Here I review key challenges and future prospects in the quest to develop a prophylactic HIV-1 vaccine. $\mathrm{t}$ has been 25 years since HIV-1 was identified as the causative agent for AIDS ${ }^{1-5}$. More than 60 million people worldwide have been infected with HIV-1, mostly in the developing world, and nearly half of these individuals have died. The development of a safe and effective HIV-1 vaccine would undoubtedly be the best solution for the ultimate control of the worldwide AIDS pandemic ${ }^{6}$, but unfortunately HIV-1 vaccine development efforts have not yet proven successful. The extraordinary diversity of HIV-1, the capacity of the virus to evade adaptive immune responses, the inability to induce broadly reactive antibody responses, the early establishment of latent viral reservoirs, and the lack of clear immune correlates of protection represent unprecedented challenges for vaccine development.

The goal of an HIV-1 vaccine would be either to prevent infection or to reduce viral loads and clinical disease progression after infection (Fig. 1). An ideal vaccine would completely block infection and provide sterilizing immunity. Although such a vaccine would be optimal, this degree of protection is not even achieved with most clinically licensed vaccines. In contrast, most licensed viral vaccines seem to function by controlling subclinical viral replication and by preventing clinical disease. It may therefore be more realistic to develop a suboptimal HIV-1 vaccine that fails to prevent infection but that provides partial immune control of viral replication after infection. Such partial control, as exemplified by a reduction in peak and setpoint viral loads after infection, has been demonstrated in certain preclinical studies by vaccines that elicit $\mathrm{T}$ lymphocyte responses.

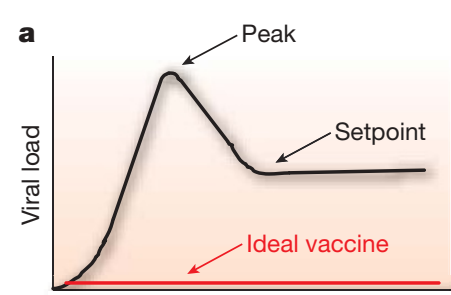

Time after infection

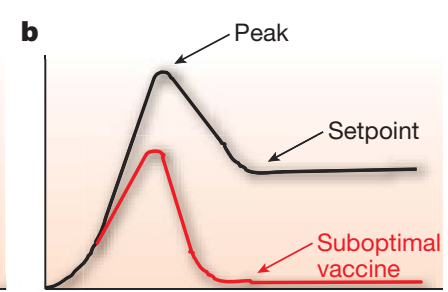

Time after infection
Figure 1 | Goals of an HIV-1 vaccine. After infection, HIV-1 replicates exponentially to a peak level and then is partially controlled to a viral setpoint level (black). a, An ideal vaccine would protect against infection and afford sterilizing immunity (red). b, A suboptimal vaccine would result in decreased peak and setpoint viral loads after infection (red).
Moreover, because viral loads represent a principal determinant of HIV-1 transmission ${ }^{7}$, it is conceivable that such a partially protective vaccine might have substantial impact on a population level.

Despite the urgent need for an HIV-1 vaccine, only two vaccine concepts have completed clinical efficacy studies so far. The first vaccine concept used monomeric HIV-1 Env gp120 protein, and the aim of this strategy was to induce Env-specific humoral immune responses. In early-phase clinical trials, gp120 immunogens elicited type-specific binding antibodies but failed to induce broadly reactive neutralizing antibodies $^{8,9}$. In two phase 3 efficacy trials sponsored by the biotechnology company VaxGen, these vaccine candidates afforded no detectable protective efficacy ${ }^{10,11}$, indicating that these type-specific antibody responses were insufficient to protect against HIV-1 infection in humans. Another phase 3 study evaluating the efficacy of a recombinant canarypox vector prime/gp120 protein boost vaccine regimen is currently underway. The second vaccine concept that has completed clinical efficacy studies involved replication-incompetent recombinant adenovirus serotype 5 (rAd5) vectors expressing HIV-1 Gag, Pol and Nef. The aim of this strategy was to elicit HIV-1-specific cellular immune responses. Early-phase clinical trials demonstrated that rAd5 vector-based vaccines elicited cellular immune responses in most subjects, although these responses were partially suppressed in individuals with pre-existing Ad5-specific neutralizing antibodies ${ }^{12}$. Phase $2 \mathrm{~b}$ efficacy trials sponsored by Merck and the National Institutes of Health (NIH) were unexpectedly terminated when the first planned interim analysis showed that this vaccine failed to protect against infection or to reduce viral loads after infection, and that vaccinees with pre-existing Ad5-specific neutralizing antibodies exhibited an enhanced rate of HIV-1 acquisition ${ }^{13}$. These results have highlighted new scientific challenges and have led to substantial debate regarding the optimal path forward for the HIV-1 vaccine field.

\section{Virologic and immunologic challenges}

The challenges in the development of a prophylactic HIV-1 vaccine are unprecedented (Box 1). The extraordinary worldwide diversity of HIV-1 presents perhaps the greatest hurdle ${ }^{14}$. Driven by the errorprone reverse transcriptase, the HIV-1 M group has diversified into nine divergent clades as well as multiple circulating recombinant forms. Amino acid sequences of Env can differ up to $20 \%$ within a particular clade and over $35 \%$ between clades $^{14,15}$. A vaccine 
Box 1 Challenges in the development of a prophylactic HIV-1 vaccine

(1) Extensive viral clade and sequence diversity.

(2) Early establishment of latent viral reservoirs.

(3) Immune correlates of protection unclear.

(4) Viral evasion of humoral and cellular immune responses.

(5) Antibody responses typically type-specific.

(6) No method exists to elicit broadly reactive neutralizing antibodies.

(7) Attenuated viruses unsafe for human use.

(8) Lack of a small-animal model.

(9) Little pharmaceutical interest.

immunogen will therefore need to contend with a remarkably high degree of viral diversity, and vaccine protection will necessarily be dependent on the capacity of immune responses to cross-react with highly heterologous viruses. Although cross-reactive humoral and cellular immune responses against conserved regions of the virus have been reported, it is reasonable to assume that protective efficacy will diminish substantially with increasing divergence between vaccine antigens and infecting viruses.

Another key challenge is the lack of clear immune correlates of protection in humans, because HIV-1-infected patients are unable to eradicate the virus. Suggestive evidence regarding immune correlates of protection might be obtained from viral challenge studies in nonhuman primates and from studies of HIV-1-infected individuals who spontaneously control viral replication to very low levels. However, definitive immune correlates of protection will probably only emerge in the context of successful vaccine efficacy studies in humans.

HIV-1-specific humoral immunity. Virus-specific neutralizing antibody titres represent key immune correlates of protection for most licensed viral vaccines, and thus early studies focused on developing HIV-1 Env subunit immunogens. Advances in our understanding of Env structure and function have begun to elucidate why generating broadly reactive neutralizing antibodies to HIV -1 by vaccination may be so difficult ${ }^{16}$. The HIV-1 Env glycoprotein is a trimer on the virion surface with extensive N-linked glycosylation that effectively shields many conserved epitopes from antibody recognition ${ }^{17,18}$. Highly immunogenic variable loops also elicit type-specific antibodies that may redirect humoral responses away from conserved regions. In addition, key conserved regions, such as the binding site of the chemokine co-receptor, are only formed after Env binds its cellular receptor CD4 and undergoes an extensive conformational change ${ }^{19}$. The development of mutations in $\mathrm{N}$-linked glycans has also been shown to lead to rapid evasion of host neutralizing antibody responses ${ }^{20,21}$.

Nevertheless, broadly reactive neutralizing antibody activity has been identified in a small number of HIV-1-infected subjects, and this reactivity seems to be largely directed against conserved regions of the Env glycoprotein such as the CD4-binding site ${ }^{22}$. The broadly reactive monoclonal antibody b12 also binds to the CD4-binding site, suggesting that this region of Env may represent a critical point of vulnerability that is potentially amenable to neutralization ${ }^{23}$. However, the CD4-binding site is recessed and only partially accessible to antibody binding. Another conserved region is the membrane-proximal external region (MPER) of gp41, which represents the target of the broadly reactive monoclonal antibodies 2F5 and 4E10. However, MPER-specific neutralizing antibodies may be difficult to elicit by vaccination for multiple reasons, including tolerance control and immunoregulation ${ }^{24}$, sequestration of the epitope in the lipid membrane ${ }^{25}$, exposure of the epitope only transiently during viral entry ${ }^{26}$, or possibly a combination of multiple factors.

The development of immunogens that induce broadly reactive neutralizing antibodies is perhaps the most important priority for the HIV-1 vaccine field ${ }^{16}$. Proof-of-concept passive transfer studies in non-human primates have shown that administration of high doses of broadly reactive monoclonal antibodies can afford sterilizing protection from infection, thus demonstrating the potential of virus-specific humoral immunity ${ }^{27,28}$. However, it has not been possible to induce such broadly reactive neutralizing antibodies by vaccination so far. Although there has been substantial progress in our understanding of Env structure and function, there are currently no vaccine candidates that are aimed at eliciting broadly reactive Envspecific neutralizing antibodies in clinical trials. It is likely that nextgeneration Env immunogens will need to be engineered antigens. Strategies that are being pursued include generating biochemically stabilized Env trimers, constraining Env immunogens in structurally defined conformations, scaffolding conserved neutralization epitopes onto foreign proteins, developing methods to circumvent immunoregulation, and designing immunogens to target specific regions such as the CD4-binding site, the MPER region and structurally conserved elements of the V3 loop. The relevance of nonneutralizing antibodies that mediate other effector functions such as antibody-dependent cell-mediated virus inhibition, complement activation and phagocytosis is also being investigated.

HIV-1-specific cellular immunity. Virus-specific T lymphocyte responses are believed to have a critical role in controlling HIV-1 replication and are therefore being actively explored in vaccine development strategies. Early studies showed that virus-specific $\mathrm{CD}^{+}{ }^{+} \mathrm{T}$ lymphocyte responses emerge during acute infection coincident with initial control of primary viremia ${ }^{29-31}$. Potent cellular immune responses have also been reported in long-term non-progressors $^{32}$, and specific HLA alleles and the breadth of Gag-specific T lymphocyte responses have been correlated with control of viral replication in HIV-1-infected individuals ${ }^{33,34}$. These data indicate the potential importance of cellular immune responses in immune control of HIV-1. Concordant with these observations, experimental depletion of $\mathrm{CD}^{+}{ }^{+}$lymphocytes has been shown to abrogate immune control of simian immunodeficiency virus (SIV) replication in rhesus monkeys ${ }^{35,36}$.

A limitation of virus-specific T lymphocyte responses is the propensity of the virus to accumulate mutations in T lymphocyte epitopes and to evade cellular immune control ${ }^{37-39}$. It is therefore likely that the breadth of epitope-specific $\mathrm{T}$ lymphocyte responses will prove critical for an HIV-1 vaccine, not only to maximize immunologic coverage of HIV-1 diversity but also to minimize the potential for viral escape from recognition by $\mathrm{T}$ lymphocytes. However, the breadth of vaccine-elicited cellular immune responses may be limited by immunodominance constraints and by the inherent tendency of $\mathrm{CD}^{+} \mathrm{T}$ lymphocyte responses to be highly focused on a limited number of epitopes.

Recent advances in the characterization of T lymphocyte responses by multiparameter flow cytometry have highlighted the functional diversity of virus-specific T lymphocytes in terms of cytokine secretion, degranulation, proliferation and other effector functions in various subpopulations of effector and memory $\mathrm{T}$ lymphocytes. It is likely that the complex functionality of T lymphocytes may ultimately prove more relevant than interferon- $\gamma$ secretion as measured by enzyme-linked immunospot (ELISPOT) assays for the evaluation of vaccine-elicited cellular immune responses. Polyfunctional T lymphocytes capable of performing multiple functions have been reported in long-term non-progressors ${ }^{40}$, in recipients of effective vaccines such as vaccinia ${ }^{41}$, and in certain preclinical challenge studies $^{42}$. These considerations suggest that the breadth ${ }^{43}$ and quality ${ }^{44}$ of $\mathrm{T}$ lymphocyte responses may prove critical in addition to the magnitude of these responses.

Perhaps the most significant limitation of vaccine-elicited cellular immune responses is that they will probably not protect against acquisition of HIV-1 infection. As a result, vaccine-induced $\mathrm{T}$ lymphocyte responses will presumably be unable to prevent lifelong infection, because the virus rapidly establishes latent reservoirs ${ }^{45,46}$. Moreover, it is unclear whether vaccine-elicited T lymphocytes will be able to function rapidly enough given that important immunopathologic events occur within the first few days of acute HIV-1 infection. HIV-1 preferentially infects HIV-1-specific $\mathrm{CD}^{+}{ }^{+} \mathrm{T}$ 
lymphocytes ${ }^{47}$ and rapidly depletes most memory $\mathrm{CD}^{+}{ }^{+} \mathrm{T}$ lymphocytes in gut-associated lymphoid tissue within the first 4-10 days of infection ${ }^{48-50}$. This sets the stage for progressive immunodeficiency as well as for chronic immune activation, which probably results at least in part from microbial translocation across damaged gastrointestinal mucosa ${ }^{51}$. Given the time required for vaccine-induced $\mathrm{CD}^{+} \mathrm{T}$ lymphocyte responses to expand after infection, it may be difficult for vaccine-elicited $\mathrm{T}$ lymphocytes to prevent these early immunopathologic events completely ${ }^{52}$.

\section{Current HIV-1 vaccine strategies}

Traditional strategies. Vaccine strategies for HIV-1 can be divided into traditional and novel vaccine approaches (Box 2). Traditional vaccine technologies include live attenuated viruses, whole killed viruses and protein subunits. Although these approaches have proven enormously successful for the development of vaccines against other viruses, they all have substantial limitations in terms of their utility for HIV-1. Live attenuated viruses have afforded substantial protective efficacy against SIV challenges in rhesus monkeys ${ }^{53,54}$, but they are unlikely to be used in humans owing to significant safety concerns $s^{55-57}$. In contrast, whole killed viruses ${ }^{58}$ and protein subunits ${ }^{10,11}$ are limited by their inability to induce broadly reactive neutralizing antibody responses as well as by their inability to elicit $\mathrm{CD}^{+} \mathrm{T}$ lymphocyte responses. Recent data, however, suggest that Toll-like receptor adjuvants may increase the utility of protein subunit immunogens $s^{59,60}$.

Novel strategies. New vaccine strategies include gene-delivery technologies such as plasmid DNA vaccines and live recombinant vectors that are engineered to express HIV-1 antigens. Plasmid DNA vaccines offer considerable promise in terms of simplicity and versatility, but multiple injections of high doses of DNA vaccines are typically required to elicit detectable immune responses in non-human primates and humans ${ }^{61,62}$. Substantial research is therefore focused on the development of adjuvants for DNA vaccines ${ }^{63,64}$ and improved delivery technologies such as in vivo electroporation ${ }^{65,66}$. Recombinant vectors include attenuated or replication-incompetent viruses, most notably adenoviruses ${ }^{12,67,68}$ and poxviruses ${ }^{69,70}$. Viral vectors, administered either alone or in the context of heterologous DNA prime/vector boost regimens, represent most HIV-1 vaccine candidates that are currently in clinical trials. Other viral vectors that are being evaluated include vesicular stomatitis virus, adeno-associated virus, Venezuelan equine encephalitis virus, cytomegalovirus, herpes simplex virus and measles virus. Bacterial and mycobacterial vectors are also being explored, including Salmonella, Listeria and Bacille Calmette-Guérin (BCG).

\section{The STEP study}

Preclinical background. Recombinant Ad5 vectors were selected for development by Merck on the basis of preclinical vector comparison studies that showed that rAd5 vectors were more immunogenic than multiple other vector modalities in rhesus monkeys ${ }^{67,71}$. Moreover, rAd5 vectors expressing SIV Gag afforded marked reductions of viral loads after challenge of rhesus monkeys with the chimaeric simianhuman immunodeficiency virus (SHIV)-89.6P (ref. 67). However, it was also observed that the same vaccine afforded minimal to no control of peak or setpoint viral loads after challenge with $\mathrm{SIV}_{\text {MAC239 }}$ (ref. 72), indicating that SIV challenges were considerably more stringent than SHIV-89.6P challenges.

\section{Box 2 | Current HIV vaccine strategies}
(1) Traditional strategies
Live attenuated viruses
Whole killed viruses
Protein subunits
(2) Novel strategies
Plasmid DNA vaccines
Live recombinant vectors

A DNA prime/rAd5 boost regimen expressing SIV Gag afforded a brief (90 days) and marginal $(0.8 \mathrm{log})$ reduction of peak viral loads after SIV $_{\text {MAC239 }}$ challenge ${ }^{72}$, but this effect was only observed in rhesus monkeys that were selected to express the major histocompatibility complex (MHC) class I molecule Mamu-A*01, which is associated with efficient virologic control ${ }^{73-75}$. A DNA prime/rAd5 boost regimen expressing multiple SIV antigens afforded increased protective efficacy in Mamu-A*01-positive rhesus monkeys ${ }^{76}$, indicating that expanding the breadth of cellular immune responses improves protection. However, neither rAd5 alone nor DNA prime/rAd5 boost regimens have been able to reduce setpoint viral loads after SIV challenge of Mamu-A*01-negative rhesus monkeys so $\operatorname{far}^{72,77}$.

Clinical studies. The Merck HIV-1 vaccine candidate was formulated as a trivalent mixture of rAd5 vectors expressing HIV-1 clade B Gag, Pol and Nef. Phase 1 clinical trials suggested that this vaccine was generally well tolerated and immunogenic in most volunteers ${ }^{12}$. However, as predicted by preclinical studies ${ }^{61}$, responses to this vaccine were partially suppressed in individuals with pre-existing neutralizing antibodies against the vaccine vector. Because $30-40 \%$ of individuals in the United States and Western Europe and 80-90\% of people in sub-Saharan Africa have pre-existing Ad5-specific neutralizing antibodies ${ }^{78-81}$, the impact of anti-vector immunity was predicted to be a limitation of rAd5 vectors.

Two phase $2 b$ 'proof-of-concept' efficacy studies were initiated by Merck and the National Institutes of Health to determine whether HIV-1-specific cellular immune responses induced by this vaccine regimen would prevent $\mathrm{HIV}-1$ infection or would reduce viral loads after infection. HIV Vaccine Trials Network (HVTN) 502, also known as the 'STEP' study, was a 3,000-subject study in the Americas, the Caribbean and Australia. HVTN 503, also called 'Phambili' (which means 'to move forward' in Xhosa), was designed as a parallel 3,000-subject study in South Africa.

On 18 September 2007, HVTN 502 was unexpectedly terminated at the first planned interim analysis when the Data and Safety Monitoring Board declared futility in the study achieving its primary end points ${ }^{13}$. Moreover, in subjects with pre-existing Ad5-specific neutralizing antibody titres, a greater number of HIV-1 infections occurred in vaccinees than in placebo recipients (Fig. 2). Although the biological basis for this observation remains unclear, these data suggest that vaccination with rAd5 vectors may be associated with an increased risk of HIV-1 acquisition in this subgroup. Post-hoc multivariate analysis further suggested that the greatest increased risk was in men who had pre-existing Ad5-specific neutralizing antibodies and who were uncircumcised.

It is currently unclear whether the lack of efficacy in the STEP study simply represents the failure of the Merck rAd5-Gag/Pol/Nef vaccine product or whether this might be the harbinger of the failure of the $\mathrm{T}$-cell vaccine concept overall. It is likely that substantial data will emerge from detailed immunologic analyses of vaccinees who subsequently became infected, and it is possible that the rAd5-Gag/Pol/ Nef vaccine failed to induce sufficient magnitude, breadth or quality of cellular immune responses ${ }^{82}$. At the present time, therefore, it would seem premature to consider the failure of this single study as the failure of T-cell-based vaccines in general.

The apparent increased risk of HIV-1 acquisition in vaccinees with pre-existing Ad5-specific neutralizing antibodies was unexpected, and this finding highlights our lack of understanding of the parameters that determine susceptibility to HIV-1 infection. The biological basis for this observation remains unclear. One hypothesis is that rAd5 vaccination of individuals with pre-existing Ad5-specific neutralizing antibodies may have resulted in potent anamnestic Ad5-specific $\mathrm{CD}^{+}{ }^{+} \mathrm{T}$ lymphocytes that were increased targets for HIV-1 infection. However, early data have suggested that Ad5-specific T lymphocyte responses after $\mathrm{rAd} 5$ vaccination are actually lower in individuals with pre-existing Ad5-specific neutralizing antibodies than in those without pre-existing Ad5-specific neutralizing antibodies (J. McElrath, 

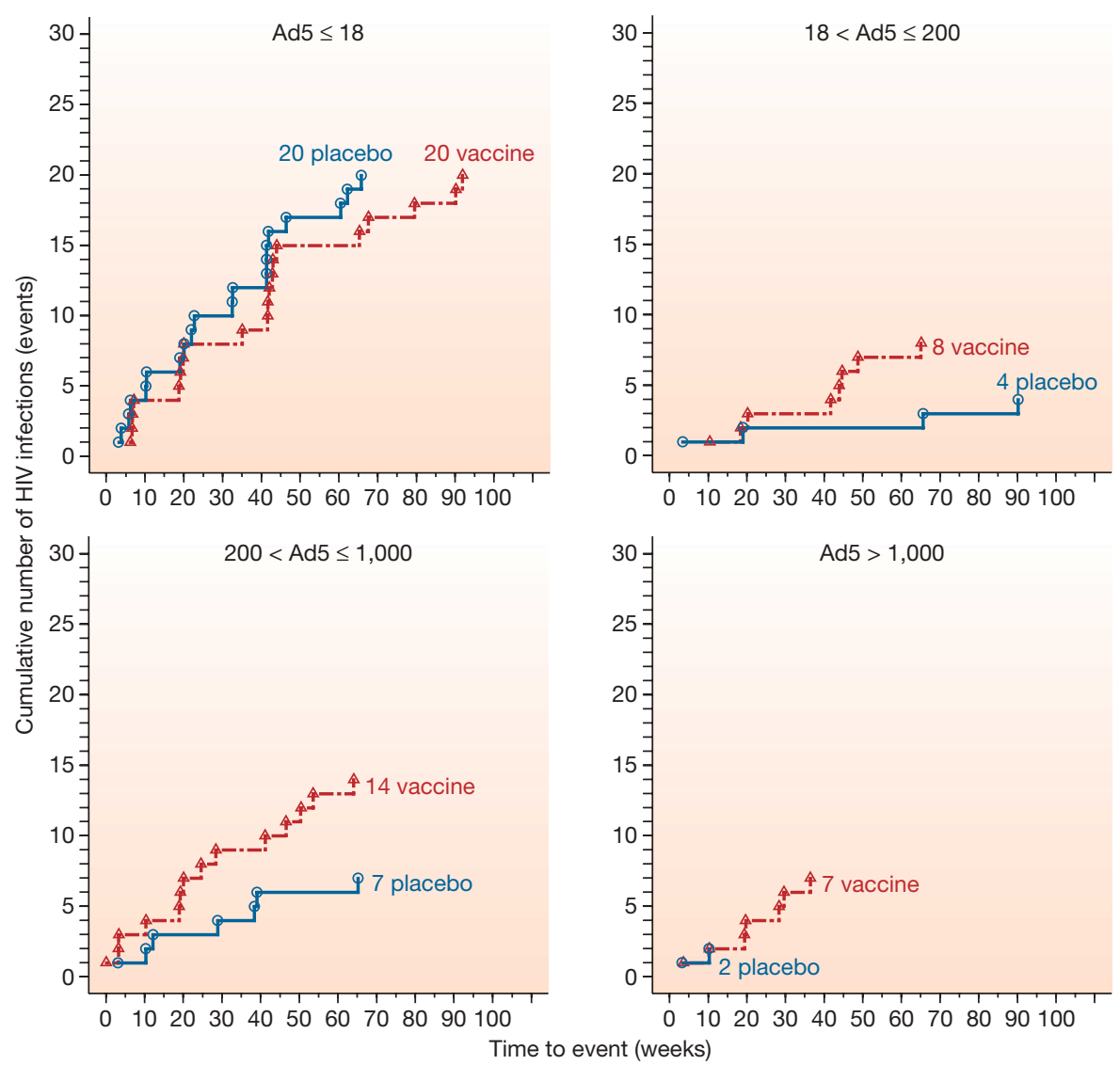

Figure 2 | Cumulative HIV-1 infections in men enrolled in the STEP study stratified by pre-existing Ad5-specific neutralizing antibody titre.

Cumulative infections as of 17 October 2007 in men enrolled in the STEP study (HVTN 502) evaluating the Merck rAd5-Gag/Pol/Nef vaccine are

unpublished observations). An alternative hypothesis is that Ad5specific neutralizing antibodies may have opsonized rAd5 vectors after immunization, resulting in altered tropism or inflammatory responses. It is also possible that pre-existing Ad5-specific neutralizing antibodies may have been a marker for other confounding variables that have not yet been identified.

\section{A STEP forward?}

Despite the disappointing results of the STEP study, several key lessons have already been learned. First, it is clear that the path forward towards an HIV-1 vaccine will be neither simple nor straightforward. Second, the importance of understanding both systemic and mucosal immune responses to vaccine vectors is paramount. Third, the biological determinants of HIV-1 acquisition and the impact that vector-specific and antigen-specific mucosal immune responses may have on this process will require intensive investigation. Fourth, clinical vaccine studies will need to adapt to the safety concerns raised by the STEP study, such as possibly excluding subjects who have preexisting neutralizing antibodies to the vaccine vector that is used until this phenomenon is more completely understood. Fifth, future T-cell-based vaccine candidates should be prioritized for clinical efficacy studies only if they are convincingly superior to the homologous rAd5-Gag/Pol/Nef regimen that has failed. Sixth, non-human primate challenge models should be recalibrated on the basis of the STEP study to guide future HIV-1 vaccine development.

The protection afforded by the homologous rAd5 regimen against SHIV-89.6P indicates that this model lacks sufficient stringency for the evaluation of T-cell-based vaccine candidates. Although the more stringent SIV challenge model cannot be considered to be validated until there is a successful clinical efficacy study in humans, it seems depicted. Infections in vaccinees (red) and placebos (blue) are shown in individuals stratified by their pre-existing Ad5-specific neutralizing antibody titres. Data represent the modified intent-to-treat population. Image courtesy of M. Robertson, Merck Research Laboratories.

reasonable to use $\mathrm{SIV}_{\mathrm{MAC239}}$ or $\mathrm{SIV}_{\mathrm{MAC} 251}$ as challenge viruses for evaluating next-generation vaccine candidates (Box 3). Preclinical challenge studies need to be adequately powered with sufficient follow-up time, and the vaccine schedule and dose should model the proposed clinical regimen. For optimal stringency, studies should exclude rhesus monkeys that express MHC class I alleles that are specifically associated with efficient virologic control, such as Mamu-A*01, Mamu-B*17 and Mamu-B*08. The use of homologous Env antigens that may inappropriately overestimate protective efficacy should also be avoided. Mucosal challenges may offer certain physiological advantages over intravenous challenges, and these challenge models should therefore be developed. Finally, increased emphasis should be placed on assessing the capacity of promising vaccine candidates to protect against highly heterologous SIV challenges, because infecting viruses in humans will almost certainly be heterologous to any vaccine sequence. Because very few heterologous SIV challenge studies have been completed so far, a practical approach may be to determine the protective efficacy of promising vaccine

Box 3 | Recommendations for preclinical challenge studies of T-cellbased vaccines

(1) Use stringent challenge virus ( SIV $_{\text {MAC239, }}$ SIV $_{\text {MAC251 }}$ ).

(2) Design study with adequate power and follow-up time.

(3) Model clinical regimen with vaccine schedule and dose.

(4) Select rhesus monkeys that lack MHC alleles associated with efficient virologic control (Mamu-A*01, Mamu-B*17, Mamu-B*08).

(5) Avoid the use of a homologous Env antigen.

(6) Assess promising vaccine concepts against both homologous and heterologous viral challenges. 
candidates against both homologous and heterologous SIV challenges. It is currently debated whether non-human primate challenge studies should be used as a formal 'gatekeeper' for advancing vaccine candidates into clinical efficacy studies, because the capacity of this model to predict the results of clinical efficacy studies remains unclear. Nevertheless, it would seem reasonable to give a relative priority to the development of vaccine candidates that lead to durable control of setpoint viral loads after SIV $_{\text {MAC239 }}$ or SIV MAC251 $_{\text {challenge. }}$

The STEP study has also had a major impact on other HIV-1 vaccine programmes in the field. HVTN 503 was terminated as it used the same rAd5-based vaccine candidate that was used in HVTN 502. The NIH Vaccine Research Center has developed a DNA prime/rAd5 boost vaccine regimen expressing clade B GagPol and multiclade Env antigens. This vaccine candidate has been shown to be immunogenic in most individuals in phase 1 studies, particularly for the Env antigens ${ }^{62,68,83}$. In preclinical studies, a DNA prime/rAd5 boost vaccine regimen expressing SIV Gag, Pol, Nef and Env antigens afforded a $1.1 \mathrm{log}$ reduction of peak viral loads for 112 days after a homologous SIV $_{\text {MAC251 }}$ challenge ${ }^{77}$. However, no durable control of setpoint viral loads was observed with this vaccine, although delayed progression to AIDS-related mortality was evident $^{77}$. NIH recently announced that it will not proceed with a large phase 2 b efficacy study known as PAVE 100, although a smaller, more focused efficacy study with this vaccine candidate is still under consideration $^{84}$. DNA prime/poxvirus boost regimens are also being evaluated using modified vaccinia Ankara $(\mathrm{MVA})^{69}$ and $\mathrm{NYVAC}^{70}$ vectors, and phase 1 clinical trials have demonstrated immunogenicity in most volunteers. Central to all of these programmes, however, is the hypothesis that DNA priming before vector boosting will improve protective efficacy. This has been observed in some $e^{72}$ but not all ${ }^{77}$ SIV challenge studies, and thus it still remains an open question that requires further investigation and should be considered a high priority.

New rAd vectors derived from Ad serotypes that are rare in human populations are also being explored as a strategy to evade pre-existing Ad5-specific neutralizing antibodies. It is hoped that such vectors may offer immunologic as well as safety advantages as compared with rAd5 vectors by circumventing pre-existing vector-specific neutralizing antibodies. However, these possibilities have not yet been confirmed in clinical trials. Current strategies include the development of rare serotype $\mathrm{rAd} 26, \mathrm{rAd} 35$ and $\mathrm{rAd} 48$ vectors ${ }^{78,79,85}$; chimaeric rAd5HVR48 vectors in which dominant Ad5-specific neutralizing antibody epitopes have been exchanged ${ }^{86}$; and non-human rAd vectors ${ }^{87,88}$. Rare serotype rAd vectors are biologically different from rAd5 vectors in terms of their cellular receptors, tropism, intracellular trafficking pathways and innate immune profiles. Moreover, rAd26 and rAd48 vectors have been shown to elicit T lymphocyte responses of a substantially different phenotype as compared with rAd5 vectors ${ }^{89}$, and potent heterologous rAd prime-boost regimens can be constructed using serologically distinct rAd vectors. We have recently demonstrated that a heterologous $\mathrm{rAd} 26$ prime/Ad5 boost regimen expressing SIV Gag afforded a durable 2.4 log reduction of setpoint viral loads after SIV MAC251 $_{1}$ challenge of Mamu-A*01-negative rhesus monkeys, whereas a homologous rAd5 regimen provided no protection in this stringent challenge mode $\mathrm{l}^{90}$. These data suggest that vaccine candidates that elicit improved magnitude, breadth and quality of T lymphocyte responses may provide superior protective efficacy as compared with homologous rAd5 regimens.

\section{Perspectives and future directions}

To a great extent, HIV-1 vaccine science is still in its infancy. Major unsolved problems remain, and a renewed commitment to basic discovery research in addition to preclinical studies and clinical trials will be required to move the field forward. Clinical trials that are focused on answering specific scientific hypotheses rather than exclusively aimed at product development may be most useful to the field at the present time. Certain vaccine regimens, such as heterologous
rAd prime-boost regimens, may offer the possibility of improved magnitude, breadth and quality of T lymphocyte responses as compared with the homologous rAd5 regimen. New antigen concepts, such as centralized consensus ${ }^{91,92}$ and mosaic ${ }^{93}$ immunogens, may also result in increased breadth of cellular immune responses and improved coverage of viral diversity.

Perhaps the most important research focus should be the development of improved Env immunogens to elicit broadly reactive neutralizing antibodies. Given the scope of this problem, increased basic research regarding the structure, function and immunogenicity of the Env glycoprotein will be required. Innovative and high-risk ideas should be pursued, and promising approaches should be tested as rapidly as possible in preclinical studies and eventually in clinical trials. Ultimately, it is likely that a combination vaccine consisting of separate vaccine components that elicit T lymphocytes and neutralizing antibodies will prove optimal. As a result, development of improved T-cell-based and antibody-based vaccine strategies should be pursued in parallel.

To achieve these goals, it will be critical to attract and to retain talented new investigators to the field. Funding programmes should therefore be expanded to encourage junior investigators to explore innovative ideas that address critical problems in the field. Given the scientific challenges currently facing the HIV-1 field, increased support and encouragement of fellows and junior faculty should be viewed as a top priority by both senior investigators and funding organizations. It will also be important for industry to continue to participate in the HIV-1 vaccine field, as biotechnology and pharmaceutical companies have critical knowledge and capacities that are not available in academia, government and non-profit organizations.

A current debate is whether the HIV-1 vaccine field can 'withstand' another vaccine efficacy study failure. For HIV-1, the scientific challenges are enormous, and thus so are the risks in testing any new vaccine concept. Clearly, the decision to advance a vaccine candidate into efficacy trials should be highly selective and based on a rigorous and transparent analysis of preclinical and clinical data. However, there is no way to determine whether a potentially promising vaccine candidate will afford protection in humans other than by conducting a clinical efficacy study. Multiple efficacy trials may be required, and many concepts will undoubtedly fail. We should therefore be ready to accept multiple failures of efficacy studies as part of the expected pathway towards the ultimate successful development of a safe and effective HIV-1 vaccine.

1. Barre-Sinoussi, F. et al. Isolation of a T-lymphotropic retrovirus from a patient at risk for acquired immune deficiency syndrome (AIDS). Science 220, 868-871 (1983).

2. Gallo, R. C. et al. Frequent detection and isolation of cytopathic retroviruses (HTLV-III) from patients with AIDS and at risk for AIDS. Science 224, 500-503 (1984).

3. Popovic, M., Sarngadharan, M. G., Read, E. \& Gallo, R. C. Detection, isolation, and continuous production of cytopathic retroviruses (HTLV-III) from patients with AIDS and pre-AIDS. Science 224, 497-500 (1984).

4. Sarngadharan, M. G., Popovic, M., Bruch, L., Schupbach, J. \& Gallo, R. C. Antibodies reactive with human T-lymphotropic retroviruses (HTLV-III) in the serum of patients with AIDS. Science 224, 506-508 (1984).

5. Schupbach, J. et al. Serological analysis of a subgroup of human T-lymphotropic retroviruses (HTLV-III) associated with AIDS. Science 224, 503-505 (1984)

6. Fauci, A. S. 25 years of HIV. Nature 453, 289-290 (2008)

7. Quinn, T. C. et al. Viral load and heterosexual transmission of human immunodeficiency virus type 1. N. Engl. J. Med. 342, 921-929 (2000).

8. Mascola, J. R. et al. Immunization with envelope subunit vaccine products elicits neutralizing antibodies against laboratory-adapted but not primary isolates of human immunodeficiency virus type 1. The National Institute of Allergy and Infectious Diseases AIDS Vaccine Evaluation Group. J. Infect. Dis. 173, 340-348 (1996).

9. Moore, J. P. et al. Primary isolates of human immunodeficiency virus type 1 are relatively resistant to neutralization by monoclonal antibodies to gp120, and their neutralization is not predicted by studies with monomeric gp120. J. Virol. 69, 101-109 (1995).

10. Flynn, N. M. et al. Placebo-controlled phase 3 trial of a recombinant glycoprotein 120 vaccine to prevent HIV-1 infection. J. Infect. Dis. 191, 654-665 (2005). 
11. Pitisuttithum, P. et al. Randomized, double-blind, placebo-controlled efficacy trial of a bivalent recombinant glycoprotein 120 HIV-1 vaccine among injection drug users in Bangkok, Thailand. J. Infect. Dis. 194, 1661-1671 (2006)

12. Priddy, F. H. et al. Safety and immunogenicity of a replication-incompetent adenovirus type 5 HIV-1 clade B gag/pol/nef vaccine in healthy adults. Clin. Infect. Dis. 46, 1769-1781 (2008)

13. Fauci, A. S. The release of new data from the HVTN 502 (STEP) HIV vaccine study. NIH News 〈http://www3.niaid.nih.gov/about/directors/news/ step_11707.htm〉 (2007)

These data demonstrate that a homologous $\mathrm{rAd5}-\mathrm{Gag} / \mathrm{Pol} / \mathrm{Nef}$ vaccine regimen did not protect against HIV-1 in humans and may have increased risk of HIV-1 acquisition in individuals with pre-existing Ad5-specific neutralizing antibodies.

14. Gaschen, B. et al. Diversity considerations in HIV-1 vaccine selection. Science 296 2354-2360 (2002)

15. Walker, B. D. \& Korber, B. T. Immune control of HIV: the obstacles of HLA and viral diversity. Nature Immunol. 2, 473-475 (2001).

16. Montefiori, D., Sattentau, Q., Flores, J., Esparza, J. \& Mascola, J. Antibody-based HIV-1 vaccines: recent developments and future directions. PLoS Med. 4, e348 (2007).

17. Kwong, P. D. et al. Structure of an HIV gp120 envelope glycoprotein in complex with the CD4 receptor and a neutralizing human antibody. Nature 393, 648-659 (1998).

18. Wyatt, R. et al. The antigenic structure of the HIV gp120 envelope glycoprotein. Nature 393, 705-711 (1998)

19. Chen, B. et al. Structure of an unliganded simian immunodeficiency virus gp120 core. Nature 433, 834-841 (2005).

20. Wei, X. et al. Antibody neutralization and escape by HIV-1. Nature 422, 307-312 (2003).

21. Richman, D. D., Wrin, T., Little, S. J. \& Petropoulos, C. J. Rapid evolution of the neutralizing antibody response to HIV type 1 infection. Proc. Natl Acad. Sci. USA 100, 4144-4149 (2003)

22. Li, Y. et al. Broad HIV-1 neutralization mediated by CD4-binding site antibodies. Nature Med. 13, 1032-1034 (2007)

23. Zhou, T. et al. Structural definition of a conserved neutralization epitope on HIV-1 gp120. Nature 445, 732-737 (2007)

24. Haynes, B. F. et al. Cardiolipin polyspecific autoreactivity in two broadly neutralizing HIV-1 antibodies. Science 308, 1906-1908 (2005).

25. Sun, Z. Y. et al. HIV-1 broadly neutralizing antibody extracts its epitope from a kinked gp41 ectodomain region on the viral membrane. Immunity 28, 52-63 (2008)

26. Frey, G. et al. A fusion-intermediate state of HIV-1 gp 41 targeted by broadly neutralizing antibodies. Proc. Natl Acad. Sci. USA 105, 3739-3744 (2008).

27. Baba, T. W. et al. Human neutralizing monoclonal antibodies of the lgG1 subtype protect against mucosal simian-human immunodeficiency virus infection. Nature Med. 6, 200-206 (2000)

28. Mascola, J. R. et al. Protection of macaques against vaginal transmission of a pathogenic HIV-1/SIV chimeric virus by passive infusion of neutralizing antibodies. Nature Med. 6, 207-210 (2000).

29. Pantaleo, G. et al. Major expansion of $\mathrm{CD}^{+} \mathrm{T}$ cells with a predominant $\mathrm{V}$ beta usage during the primary immune response to HIV. Nature 370, 463-467 (1994)

30. Koup, R. A. et al. Temporal association of cellular immune responses with the initial control of viremia in primary human immunodeficiency virus type 1 syndrome. J. Virol. 68, 4650-4655 (1994)

31. Borrow, P., Lewicki, H., Hahn, B. H., Shaw, G. M. \& Oldstone, M. B. Virus-specific $\mathrm{CD}^{+}$cytotoxic T-lymphocyte activity associated with control of viremia in primary human immunodeficiency virus type 1 infection. J. Virol. 68, 6103-6110 (1994).

32. Musey, L. et al. Cytotoxic-T-cell responses, viral load, and disease progression in early human immunodeficiency virus type 1 infection. N. Engl. J. Med. 337, 1267-1274 (1997)

33. Kiepiela, P. et al. Dominant influence of HLA-B in mediating the potential coevolution of HIV and HLA. Nature 432, 769-775 (2004).

34. Kiepiela, P. et al. CD8 ${ }^{+} \mathrm{T}$-cell responses to different HIV proteins have discordant associations with viral load. Nature Med. 13, 46-53 (2007).

35. Schmitz, J. E. et al. Control of viremia in simian immunodeficiency virus infection by $\mathrm{CD}^{+}$lymphocytes. Science $283,857-860$ (1999)

36. Jin, $X$. et al. Dramatic rise in plasma viremia after $\mathrm{CD}^{+} \mathrm{T}$ cell depletion in simian immunodeficiency virus-infected macaques. J. Exp. Med. 189, 991-998 (1999).

37. Phillips, R. E. et al. Human immunodeficiency virus genetic variation that can escape cytotoxic T cell recognition. Nature 354, 453-459 (1991).

38. Allen, T. M. et al. Tat-specific cytotoxic T lymphocytes select for SIV escape variants during resolution of primary viraemia. Nature 407, 386-390 (2000).

39. Barouch, D. H. et al. Eventual AIDS vaccine failure in a rhesus monkey by viral escape from cytotoxic T lymphocytes. Nature 415, 335-339 (2002).

40. Betts, M. R. et al. HIV nonprogressors preferentially maintain highly functional HIV-specific CD8 ${ }^{+}$T cells. Blood 107, 4781-4789 (2006).

41. Precopio, M. L. et al. Immunization with vaccinia virus induces polyfunctional and phenotypically distinctive $\mathrm{CD}^{+}{ }^{+} \mathrm{T}$ cell responses. J. Exp. Med. 204, 1405-1416 (2007).

42. Darrah, P. A. et al. Multifunctional TH1 cells define a correlate of vaccinemediated protection against Leishmania major. Nature Med. 13, 843-850 (2007).
43. Watkins, D. I., Burton, D. R., Kallas, E. G., Moore, J. P. \& Koff, W. C. Nonhuman primate models and the failure of the Merck HIV-1 vaccine in humans. Nature Med. 14, 617-621 (2008)

44. Seder, R. A., Darrah, P. A. \& Roederer, M. T-cell quality in memory and protection: implications for vaccine design. Nature Rev. Immunol. 8, 247-258 (2008).

45. Chun, T. W. et al. Quantification of latent tissue reservoirs and total body viral load in HIV-1 infection. Nature 387, 183-188 (1997).

46. Chun, T. W. et al. Early establishment of a pool of latently infected, resting CD4 ${ }^{+}$ cells during primary HIV-1 infection. Proc. Natl Acad. Sci. USA 95, 8869-8873 (1998).

47. Douek, D. C. et al. HIV preferentially infects HIV-specific CD4 ${ }^{+} \mathrm{T}$ cells. Nature 417, 95-98 (2002)

48. Veazey, R. S. et al. Gastrointestinal tract as a major site of CD4 ${ }^{+} \mathrm{T}$ cell depletion and viral replication in SIV infection. Science 280, 427-431 (1998).

49. Mattapallil, J. J. et al. Massive infection and loss of memory $C D 4^{+} \mathrm{T}$ cells in multiple tissues during acute SIV infection. Nature 434, 1093-1097 (2005).

50. Li, Q. et al. Peak SIV replication in resting memory $\mathrm{CD}^{+}{ }^{+} \mathrm{T}$ cells depletes gut lamina propria CD4 ${ }^{+}$T cells. Nature 434, 1148-1152 (2005).

51. Brenchley, J. M. et al. Microbial translocation is a cause of systemic immune activation in chronic HIV infection. Nature Med. 12, 1365-1371 (2006).

52. Mattapallil, J. J. et al. Vaccination preserves CD4 memory T cells during acute simian immunodeficiency virus challenge. J. Exp. Med. 203, 1533-1541 (2006).

53. Daniel, M. D., Kirchhoff, F., Czajak, S. C., Sehgal, P. K. \& Desrosiers, R. C. Protective effects of a live attenuated SIV vaccine with a deletion in the nef gene. Science 258, 1938-1941 (1992)

54. Wyand, M. S., Manson, K. H., Garcia-Moll, M., Montefiori, D. \& Desrosiers, R. C. Vaccine protection by a triple deletion mutant of simian immunodeficiency virus. J. Virol. 70, 3724-3733 (1996).

55. Learmont, J. C. et al. Immunologic and virologic status after 14 to 18 years of infection with an attenuated strain of HIV-1. A report from the Sydney Blood Bank Cohort. N. Engl. J. Med. 340, 1715-1722 (1999).

56. Baba, T. W. et al. Pathogenicity of live, attenuated SIV after mucosal infection of neonatal macaques. Science 267, 1820-1825 (1995)

57. Baba, T. W. et al. Live attenuated, multiply deleted simian immunodeficiency virus causes AIDS in infant and adult macaques. Nature Med. 5, 194-203 (1999)

58. Murphey-Corb, M. et al. A formalin-inactivated whole SIV vaccine confers protection in macaques. Science 246, 1293-1297 (1989).

59. Wille-Reece, U. et al. HIV Gag protein conjugated to a Toll-like receptor $7 / 8$ agonist improves the magnitude and quality of Th1 and $\mathrm{CD} 8^{+} \mathrm{T}$ cell responses in nonhuman primates. Proc. Natl Acad. Sci. USA 102, 15190-15194 (2005).

60. Wille-Reece, $U$. et al. Toll-like receptor agonists influence the magnitude and quality of memory $T$ cell responses after prime-boost immunization in nonhuman primates. J. Exp. Med. 203, 1249-1258 (2006)

61. Casimiro, D. R. et al. Comparative immunogenicity in rhesus monkeys of DNA plasmid, recombinant vaccinia virus, and replication-defective adenovirus vectors expressing a human immunodeficiency virus type 1 gag gene. J. Virol. 77, 6305-6313 (2003)

62. Graham, B. S. et al. Phase 1 safety and immunogenicity evaluation of a multiclade HIV-1 DNA candidate vaccine. J. Infect. Dis. 194, 1650-1660 (2006).

63. Barouch, D. H. et al. Control of viremia and prevention of clinical AIDS in rhesus monkeys by cytokine-augmented DNA vaccination. Science $290,486-492$ (2000).

64. Chong, S. Y. et al. Comparative ability of plasmid IL-12 and IL-15 to enhance cellular and humoral immune responses elicited by a SIVgag plasmid DNA vaccine and alter disease progression following SHIV(89.6P) challenge in rhesus macaques. Vaccine 25, 4967-4982 (2007).

65. Luckay, A. et al. Effect of plasmid DNA vaccine design and in vivo electroporation on the resulting vaccine-specific immune responses in rhesus macaques. J. Virol. 81, 5257-5269 (2007)

66. Liu, J., Kjeken, R., Mathiesen, I. \& Barouch, D. H. Recruitment of antigenpresenting cells to the site of inoculation and augmentation of human immunodeficiency virus type 1 DNA vaccine immunogenicity by in vivo electroporation. J. Virol. 82, 5643-5649 (2008).

67. Shiver, J. W. et al. Replication-incompetent adenoviral vaccine vector elicits effective anti-immunodeficiency-virus immunity. Nature 415, 331-335 (2002)

68. Catanzaro, A. T. et al. Phase 1 safety and immunogenicity evaluation of a multiclade HIV-1 candidate vaccine delivered by a replication-defective recombinant adenovirus vector. J. Infect. Dis. 194, 1638-1649 (2006).

69. Amara, R. R. et al. Control of a mucosal challenge and prevention of AIDS by a multiprotein DNA/MVA vaccine. Science 292, 69-74 (2001).

70. Harari, A. et al. An HIV-1 clade C DNA prime, NYVAC boost vaccine regimen induces reliable, polyfunctional, and long-lasting $T$ cell responses. J. Exp. Med. 205, 63-77 (2008)

71. Shiver, J. W. \& Emini, E. A. Recent advances in the development of HIV-1 vaccines using replication-incompetent adenovirus vectors. Annu. Rev. Med. 55, 355-372 (2004).

72. Casimiro, D. R. et al. Attenuation of simian immunodeficiency virus SIVmac239 infection by prophylactic immunization with DNA and recombinant adenoviral vaccine vectors expressing Gag. J. Virol. 79, 15547-15555 (2005).

This manuscript demonstrates that homologous $\mathrm{rAd5}$ vaccine regimens were minimally effective against SIV $_{\text {MAC239 }}$ challenges in rhesus monkeys. 
73. Mothe, B. R. et al. Expression of the major histocompatibility complex class I molecule Mamu-A*01 is associated with control of simian immunodeficiency virus SIVmac239 replication. J. Virol. 77, 2736-2740 (2003).

74. Pal, R. et al. ALVAC-SIV-gag-pol-env-based vaccination and macaque major histocompatibility complex class I $(A * 01)$ delay simian immunodeficiency virus SIVmac-induced immunodeficiency. J. Virol. 76, 292-302 (2002).

75. Zhang, Z. Q. et al. Mamu-A*01 allele-mediated attenuation of disease progression in simian-human immunodeficiency virus infection. J. Virol. 76, 12845-12854 (2002).

76. Wilson, N. A. et al. Vaccine-induced cellular immune responses reduce plasma viral concentrations after repeated low-dose challenge with pathogenic simian immunodeficiency virus SIVmac239. J. Virol. 80, 5875-5885 (2006).

77. Letvin, N. L. et al. Preserved $\mathrm{CD}^{+}{ }^{+}$central memory T cells and survival in vaccinated SIV-challenged monkeys. Science 312, 1530-1533 (2006).

78. Vogels, R. et al. Replication-deficient human adenovirus type 35 vectors for gene transfer and vaccination: efficient human cell infection and bypass of preexisting adenovirus immunity. J. Virol. 77, 8263-8271 (2003).

79. Abbink, P. et al. Comparative seroprevalence and immunogenicity of six rare serotype recombinant adenovirus vaccine vectors from subgroups $B$ and $D$. J. Virol. 81, 4654-4663 (2007).

80. Thorner, A. R. et al. Age dependence of adenovirus-specific neutralizing antibody titers in individuals from sub-Saharan Africa. J. Clin. Microbiol. 44, 3781-3783 (2006).

81. Kostense, S. et al. Adenovirus types 5 and 35 seroprevalence in AIDS risk groups supports type 35 as a vaccine vector. AIDS 18, 1213-1216 (2004).

82. Fauci, A. S. et al. HIV vaccine research: the way forward. Science 321, 530-532 (2008).

This perspective describes revised $\mathrm{NIH}$ research priorities for HIV-1 vaccine research.

83. Catanzaro, A. T. et al. Phase I clinical evaluation of a six-plasmid multiclade HIV-1 DNA candidate vaccine. Vaccine 25, 4085-4092 (2007).

84. Fauci, A. S. NIAID will not move forward with the PAVE 100 HIV vaccine trial. NIH News 〈http://www3.niaid.nih.gov/news/newsreleases/2008/pave100.htm〉 (2008).
85. Barouch, D. H. et al. Immunogenicity of recombinant adenovirus serotype 35 vaccine in the presence of pre-existing anti-Ad5 immunity. J. Immunol. 172 6290-6297 (2004).

86. Roberts, D. M. et al. Hexon-chimaeric adenovirus serotype 5 vectors circumvent pre-existing anti-vector immunity. Nature 441, 239-243 (2006).

87. Farina, S. F. et al. Replication-defective vector based on a chimpanzee adenovirus. J. Virol. 75, 11603-11613 (2001)

88. Fitzgerald, J. C. et al. A simian replication-defective adenoviral recombinant vaccine to HIV-1 gag. J. Immunol. 170, 1416-1422 (2003).

89. Liu, J. et al. Magnitude and phenotype of cellular immune responses elicited by recombinant adenovirus vectors and heterologous prime-boost regimens in rhesus monkeys. J. Virol. 82, 4844-4852 (2008)

90. Barouch, D. H. Novel adenovirus vector-based vaccines for HIV-1. Keystone Symposia on HIV Vaccines, Banff, Canada. Abstract X7 009, page 60 (Keystone Symposia, 27 March-1 April 2008)

91. Liao, H. X. et al. A group M consensus envelope glycoprotein induces antibodies that neutralize subsets of subtype B and C HIV-1 primary viruses. Virology 353, 268-282 (2006).

92. Weaver, E. A. et al. Cross-subtype T-cell immune responses induced by a human immunodeficiency virus type 1 group $m$ consensus env immunogen. J. Virol. 80, 6745-6756 (2006).

93. Fischer, W. et al. Polyvalent vaccines for optimal coverage of potential T-cell epitopes in global HIV-1 variants. Nature Med. 13, 100-106 (2007). This manuscript proposes the use of polyvalent 'mosaic' antigens to improve immunologic coverage of global HIV-1 diversity.

Acknowledgements The author would like to thank R. Dolin, N. Letvin, J. Mascola and J. McElrath for critically reviewing this manuscript. The author acknowledges support from the National Institutes of Health and the Bill \& Melinda Gates Foundation.

Author Information Reprints and permissions information is available at www.nature.com/reprints. Correspondence and requests for materials should be addressed to D.H.B. (dbarouch@bidmc.harvard.edu). 